\title{
A Survey of Communications and Collaborative Web Technologies
}

\author{
Narayanan Kulathuramaiyer ${ }^{1}$ and Hermann Maurer ${ }^{2}$ \\ ${ }^{1}$ Faculty of Computer Science and Information Technology, University Malaysia Sarawak, Malaysia \\ ${ }^{2}$ Institute for Information Systems and Computer Media (IICM), Graz University of Technology, Austria
}

\begin{abstract}
The web has changed the way we do things in a variety of ways. It is not uncommon to associate the term disruptive technologies to the radical changes happening on the web. This paper takes an encompassing view of developments surrounding the web with a particular emphasis on emerging trends and evolving application scenarios. We provide a broad overview of technologies, in revealing directions for emerging developments as well as directions for next-generation developments. This survey paper first reviews aspects of the web growth and its contributing factors such as the expanding landscape of social networks, mass collaborative system capabilities, and eCommunities. We then present concerns relating to the numerous developments, particularly with respect to social and political developments. This paper clearly points out the knowledge gaps and the ensuing dangers arising from the purely commercial perspectives and motivation that is driving the web. By focusing on the trees rather than the forest as a whole, we are in the process of losing ourselves and endangering our future. Subsequently, we explore the implications of current developments in enabling the web to serve as a platform for serious work, in allowing users to focus on creatively shaping of our world.
\end{abstract}

Keywords: web technologies, web services, web applications, social implications, dangers of the emerging technologies

Categories: H.4., I.2., K.4.

\section{Introduction}

The web has truly transformed the way we do things in a variety of ways. For many of us, it now becomes difficult to imagine a world without the web. It has grown to become an immense resource beyond anything we have ever seen before. For the younger generations, it is becoming the preferred platform for collaborative problem solving and fast-and-easy decision-making. Due to their increasing faith and reliance on this technology, we see the need for the responsible delivery of information that actually address the knowledge needs, while seriously taking into consideration the long-term implications and consequences on society.

While the web is establishing itself as an increasingly engaging social platform that is connecting and empowering communities of users and individuals, there are clear warning signals indicating that it is producing the dumbest generation ever [Maurer, 2015]. As emerging developments strives to assimilate everything we do within the folds of the web, serious interventions are abruptly needed to set things straight. This survey paper considers technological development in a broad sense, while attempting to provide an overview of emerging developments and trends. Emerging technological directions are contextualized within an encompassing framework that projects a converging viewpoint in spite of all the diverse, isolated explorations. The main focus however will be on concerns surrounding the web and its emerging applications.

The first section of this paper attempts to provide an overview of evolutionary web developments. Here we provide a broad overview of the aspects such as social networks, eCommunities and mass collaboration systems. Subsequently, we will review the layering framework of technologies and explore various concerns. 


\section{The Evolution of the Web}

\subsection{Web 2.0, 3.0 and Beyond}

In considering the evolutionary development of the web, a discussion on Web 2.0, and Web 3.0 nomenclature is seen to be relevant. Though it is not our intention to go into definitions relating to a linear progressions of the web, their aspects need to be presented as background.

Barassi and Treré (2012) describe Web 1.0 technologies as those which enable the cognitive processes of communication. It is mainly seen as a user's engagement with hypertext. This read-only web tends to describe the early phase of web. Web 2.0 technologies on the other hand relate to communicative processes, which have been enabled by interactive platforms [Barassi and Treré, 2012]. Web 2.0 is seen here as a paradigm that has brought about innumerable possibilities of democratization for engaging and empowering individuals (see [Gillmor, 2006]). It can also be seen as the stage when the internet's communication facilities become integrated with the web to enable sophisticated user-centered communication mechanisms. In this respect, Web 3.0 is then described as a facilitator of cooperative processes that allows the collaborative creation of new meaning [Barassi and Treré, 2012]. Though the boundaries between these stages may not be obvious, we now see the emergence of numerous cooperative applications that are enhancing user experience and enabling a wide range of new services.

An outcome of these marked rapid developments as seen in the emergence of the Social $\mathrm{Web}^{1}$, cuts across the whole web allowing people to form relationships with each other. This Social Web has connected everyone in the world, either directly or indirectly. It has to be noted that development of the web has already affected all of us, regardless of whether we are physically connected or not.

The deep web is also another fascinating development. The size of this invisible web is estimated to be 500 times larger than the web as we know it. Through this deep web, many documents are being kept away from regular users; there are also many activities that are kept away from the main stream of web activities. This includes the much-talked about Bitcoin currency [Bitcoin, 2008] and its entirely decentralized financial system. There are also syndicates operating within the regions of the deep web where anonymity is said to still exist. Though the discussions of the deep web are beyond the scope of this paper, we note here the open door available for explorations into various untapped potentials.

\subsection{The Web as a Landscape of Emerging Global Communities}

The impetus for the web's growth is in its unifying ability in tying together people, processing power and knowledge. The landscape of the global leaders are shaping the new world order dominated by companies from the United States and China [Matranga, 2013]. Emerging monopolies are taking shape [Kulathuramaiyer \& Balke, 2007] as promising startups tend to be bought by the market leaders such as Google (YouTube, Google+, Orkut), Facebook (WhatsApp) and Yahoo (Tumblr). The leading social media sites, Facebook and YouTube, have over a billion monthly active users, while emerging sites are surpassing 20 million users, with some reaching 350 million users. The symbolic power associated with these developments has only been partially addressed in [Kulathuramaiyer \& Balke, 2007].

Many of these emerging sites such as Google+, Tumblr, and Pinterest, are mainly expanding through large-scale content curation, aggregation and re-purposing. A massive number of postings together with a continuous stream of remixing or re-blogging (legally allowed with a proper attribution and linking to original sites) shapes the dynamicity of these sites. The reblogging of a single post in Tumblr was shown to have over 60,000 notes and over $35,000 \mathrm{sec}$ ond level re-bloggings during the Golden Globe awards in 2013 [UnionMetric, 2013]. In one month alone, Twitter and Tumblr together generated over 3 million postings and re-postings on this event only [UnionMetric, 2013]. Though Twitter has played an important role in spreading information during catastrophes and emer-

\footnotetext{
${ }^{1}$ http://www.w3.org/2005/Incubator/socialweb/XGR-socialweb-20101206/
} 
gency situations, it is also a dominant, compulsive engaging platform for spreading trivialities.

\subsection{The Web as a Connected Small World}

The cultivation of egocentric networks serves as a fundamental building block for massively large social networks. It is now possible to map the social linkages of an individual in conjunction with other personal information as either provided by the users or even dynamically discovered based on usage tracking. This visualization, as can be shown in Figure $1^{2}$, provides the ability for a profiling of users in a global environment. With 291 first level connections, the paid service of LinkedIn would allow a connection to over 5 million individuals within 34 levels of connections. This contrast in the number of connections that become available contextualizes each user's contribution within a global space of 100 million users.

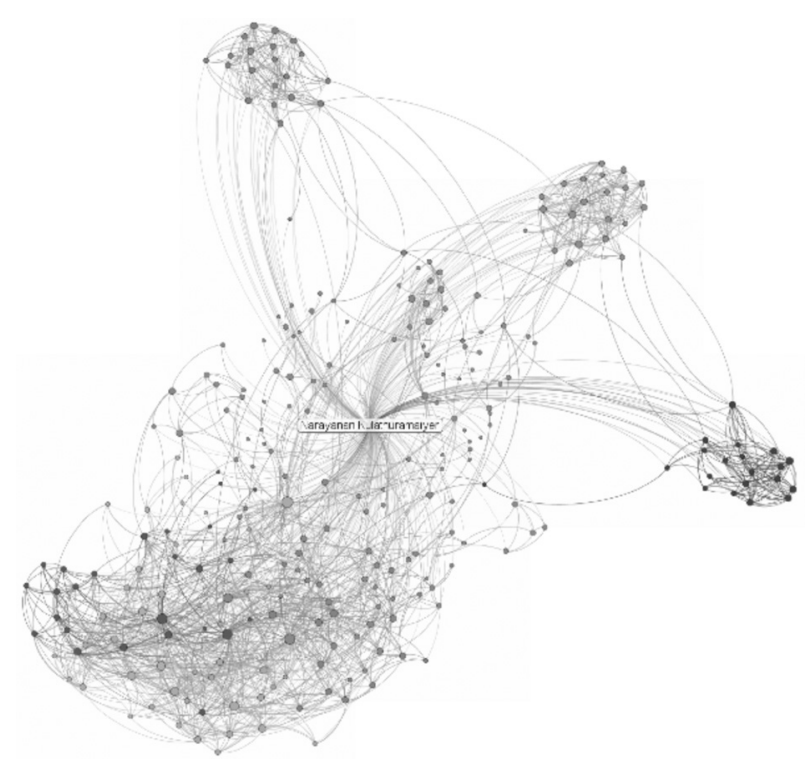

Figure 1. Personal social network visualization.

These massive networks serve as valuable resources whereby user profiling and identity management become key aspects in modelling user activities within conjoined spaces of personal and professional connections.

[Zhong, et al., 2014] indicates that emerging sites such as Pinterest expand their own networks aggressively by using social bootstrapping measures. Facebook's contacts networks are being copied in producing a gigantic connected network for these companies [Zhong, et al., 2014]. Users help these networks grow by consenting to invite their contacts (e.g. Facebook friends, Gmail contacts) to become registered users of these sites. Such an approach has a significant impact on the growth of emerging social networks [Zhong, et al., 2014]. This bootstrapping allows these social networks to become able to link and expand user clusters augmented with further defining interconnections.

With a reliable mass of individuals, these sites are able to harness the macroscopic network structure to gain insights into their extensive user base. They are further able to harness the small world ${ }^{3}$ phenomenon [Travers \& Milgram, 1969], which has now been demonstrated on a number of social networks. The small world also relates to the notion of 6 degrees of separa$t_{i o n}{ }^{4}$. By employing these convergence structures, social network sites acquire the ability to directly connect users in order to unify people for achieving a common course. Such a symbolic power could well be applied to drive communities for a global-scale mobilization of people. State-of-the-art intelligent collaborative filtering techniques model clusters of connected individuals based on multi-dimensional user profiles. These closely tied clusters of users can then be subject to manipulation for private gains, surveillance or abusive purposes as will be discussed in Section 4.

The small world phenomenon can be illustrated for a scholarly network as shown in Figure $2^{5}$. The second author is two degrees apart from Paul Erdos, while the first author as his coauthor is three degrees apart. Some individuals

\footnotetext{
${ }^{2}$ Linkedin API has been used as a Web Service to visualise this network. The service called InMaps has however been disabled by LinkedIn. http://help.linkedin.com/app/answers/detail/a_ id/4949//inmaps---no-longer-supported

${ }^{3}$ A visualization of the experiment can be found in http://en.wikipedia.org/wiki/Small-world_ experiment

4 The Erdos Number Project is described here http://www.oakland.edu/enp/. It states that the separation between any two mathematicians is at most 6 in a co-author networks. The distance between the second author and Paul Erdos is calculated as 3, based on the tool provided by the American Mathematical Society, http://www.ams.org/mathscinet/collaborationDistance.html

5 This network was built using Microsoft Academic Search network for the authors.
} 


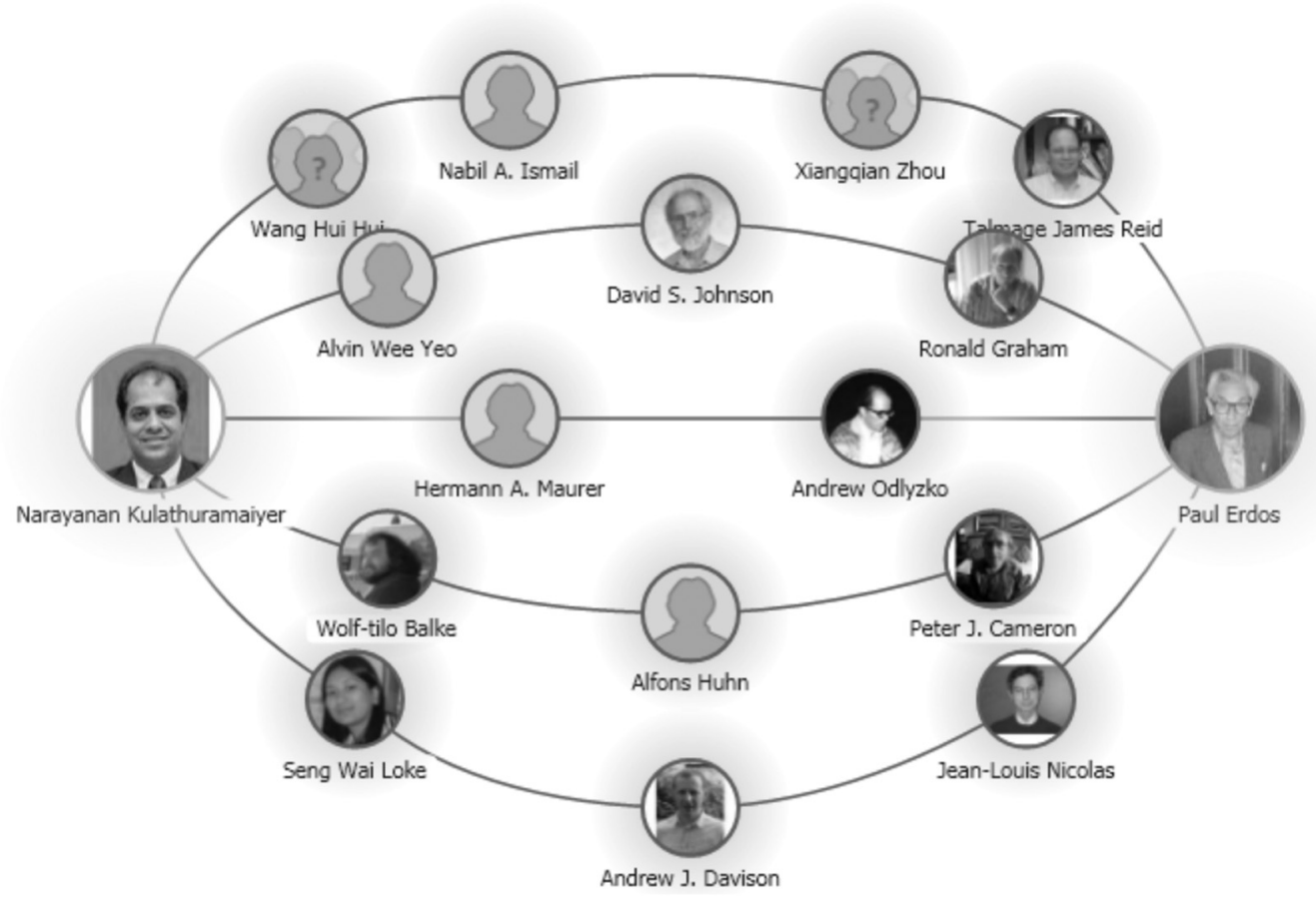

Figure 2. Small world phenomenon.

as such can be identified as high-degree connectors or hubs for linking together other members of associated network clusters. These individuals could then be subjected to targeted profiling to effectively influence the lives of connected clusters.

These developments open up a platform for large-scale experimentations that model global developments resulting from localized user strategy and behaviour [Kearns, 2012]. At the same time, these insightful connections are ideal resources for exploitation in the hands of surveillance agencies and saboteurs. Even if these links don't pose any danger to us right now, one has to remember the permanence of information on the web, which could strike back at a later time in our lives.

The rapid growth of user networks and the increasing connecting ability can at times become a problem to users. Superficial connections (loosely connected links), if not strictly managed, may expand and become expressed much faster than one may be able to handle. Even worse, the networks could include incompatible personalities or malicious users waiting for a chance to exploit susceptible users. Visualization of the layers of the community that a user typically engages in is shown in Figure 3.
The layered model of eCommunity systems [Lid, 2010] can be used as the basis for defining of relationship patterns in egocentric networks. The aspect of support groups has to be considered in the design of community management strategy. It has to be noted that people are only able to service active connections with a limited number of persons within an engaged community [Goncalves, et al., 2011] [Reisinger, 2010]. As such, users will then need effective community management strategy or adjust levels of

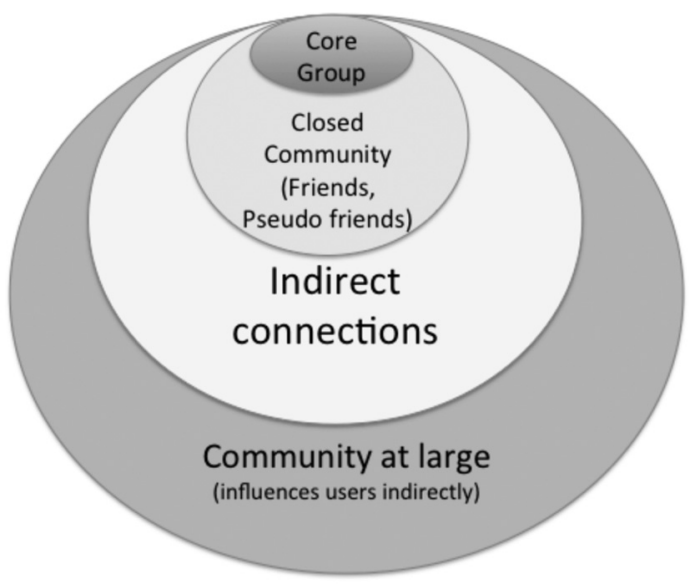

Figure 3. Layered user engagement in eCommunities. 
coupling and interaction with users to maintain and benefit from meaningful relationships.

Furthermore, the entire landscape of social networking sites has to be seen as a whole in illustrating the extent of user engagement, in shaping the overall experience of users. This experience takes shape as users switch between communities of users playing a variety of different roles, with rapid context switching between tasks [Wenger, 2009]. [Wenger, 2010] describes the trajectory of learning that happens across a landscape of communities of practice. Web applications will then need to consider thresholds on community size which directly corresponds to the quality of resulting interactions and collaborative efforts.

\section{Cross-Cutting View of Web Enabling Technologies}

Unlike studies that merely focuse on technical aspects or on social aspects alone, tending to take a narrow view of web technologies and applications, we take a more encompassing view. Having taken a microscopic view of social networks in the previous Section, we now present an overarching perspective of technological developments.

The discussions in this sections will provide a broad and encompassing overview of web services and web technologies in shedding light on emerging applications. Figure 4 combines the modelling and harnessing of user spaces and contributions for producing powerful platforms that are enabling state-of-the-art applications.

Sections 3.1 and 3.2 first review the framework and the emerging developments. Section 3.3 then describes the engagement of user modelling, context-awareness and profiling in paving the way for mass collaborative systems. Section 3.4 illustrates the slightly disconnected discussion on applications, as such an integration framework has not been used as a guiding principle.

\subsection{Overview of the Layering Framework}

A large amount of research has been centered around standards and layered framework for the enabling of web technologies. These works tend to be linked to web services, which serves as the foundation for collaborative applications

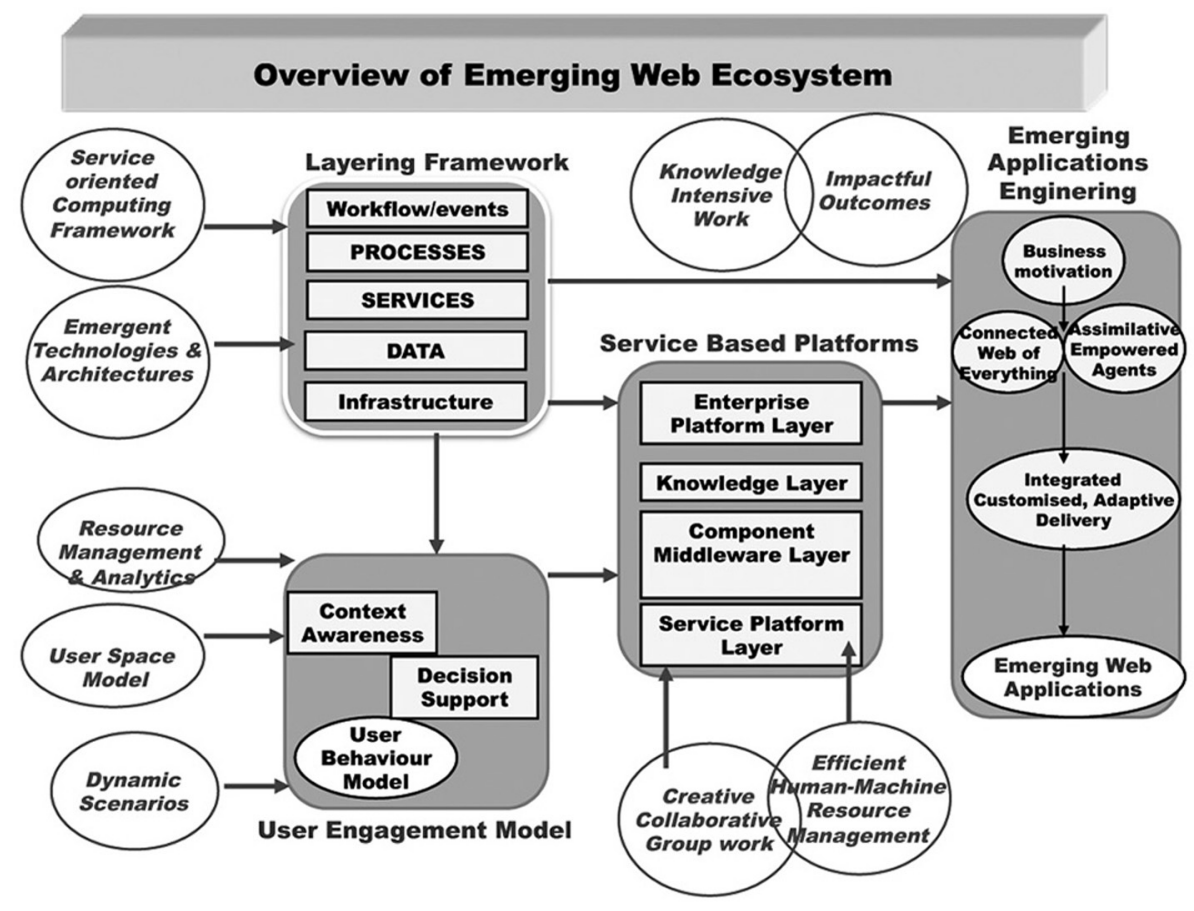

Figure 4. Overview of emerging web ecosystems. 
and as coordination mechanisms between distributed initiatives. Service orientation as a viewpoint, sees everything from platforms, processing power and applications as components that can be remixed and expanded in scope and capability in a bigger frame of things.

In summary, the web relies on a service oriented architecture and related frameworks and service oriented computing in producing service-based applications [Di Nitto, et al., 2008] and serviceoriented enterprises [Steen, et al., 2005]. Arising from the flexible framework that has constructed aspects such as the platform, processing and computing powers can be acquired and expanded as required for the emerging developments and expanded provisioning of high performance computing. Additionally, resources can include special purpose servers, grids, crowdsourced human computation resources, architecture, software components and aggregated applications.

\subsection{Emerging Technologies}

As the layering of technology components is evolving in an ad-hoc manner, defacto standards are emerging. We present here related recent developments.

Complex analysis pipelines combining distributed web services with data and tools are becoming available as workflows in specific domain areas. In the area of bio-informatics, scientists and researchers now have access to over [Hull, et al., 2006] 1000 tools and resources. The workflows can then be shared, reused and re-purposed to suit the need of researchers.

In 2008, the number of things connected to the internet exceeded that of the world population [Cisco ISBG, 2011], and the number is expected to reach 50 billion connected things by 2020 . The current view of closed groups of devices connected by gateway models will have to give way to a much more scalable one. Social networks and social web will then have a more important role to play in the emerging social web of things and Social Internet of Things (SIoT) [Iera, et al., 2014].

The SIoT promises the advent of connected intelligent devices, addressing concerns of navigability and trust. Research directions are exploring both the expansion of existing social networks to accommodate the explosive development of connected things such as in evrythng.com and also the emerging social tools that allow people to connect to physical and virtual objects, things, services and APIs such as Paraimpu [Iera, et al., 2014]. At the same time, composer tools and personalized tools are emerging. Other developments include the context aware middleware for IoT.

These emerging developments will need to tie in developments of the social networks, as described earlier in a more seamless way, in a way that is able to integrate personal aspects.

\subsection{User Engagement and Modelling Mass Collaboration}

The study of human-computer inter-relationships arising from interaction, feedback and bidirectional influence has to be considered together with expanding technological advancements. The engagement of users, user modelling, context-awareness and profiling are all aspects that need to be studied together, to ensure user centric view of emerging systems.

In this respect, a key development of the web is the emergence of mass collaboration systems (MCS). A MCS enlists a large number of human agents as a means to solve a variety of problems [Doan, et al., 2011]. Here, the notion of collaboration can either be implicit (such as Duolingo $^{6}$ ) or explicit (such as Wikipedia).

Successful platforms such as TopCoder ${ }^{7}$ make use of a divide and conquer approach where complex problems are broken down into manageable chunks and then broadcast to a large crowd of users. These sites $\left(Q^{2}{ }^{2}{ }^{8}{ }^{8}{ }^{8}\right.$, Innocentive $^{9}$, TopCoder) run competitions, and, as a result, are able to identify individuals with

\footnotetext{
6 www.duolingo.com

7 http://www.topcoder.com/

${ }^{8}$ https : //www. quirky.com/

${ }^{9}$ https://www. innocentive.com
} 
a vast talent and expertise and a willingness to explore and provide creative solutions. The management of such expertise in a systematical way to engage them in a collaborative problemsolving for complex problems remains an open issue [Kittur, et al., 2011].

It has been noted that these models are limited in terms of cooperation and coordination among users, particularly when it involves illdefined or complex problems with interdependent parts [Kittur, et al., 2011]. This is particularly true when it involves a large number of participants or contributors. The study of MCS, cannot be undertaken without a deep understanding of community participation levels. Community participation and engaging of users as partners [Zaman, et al., 2013] are important in the design of human-centric crowd systems [Kittur, et al., 2013].

\subsection{Service Oriented Platforms}

The idea of a programmable web enabled by service mashups has been seen as a promising direction. The developments of mashups or integrated applications that make use of publically available APIs has not, as anticipated, enabled a truly programmable web. Despite the steady growth of APIs, there are gaps between high-level specification and the 'wired' programmable components.

Web service composition is a heavily researched area. Concerns with this area include interoperability, ubiquity and personalization of composite services [Sheng et al., 2014]. Web service composition/integration can be using two main approaches: the integration of complex enterprise applications, or the integration of largescale distributed hypermedia systems.

From the composition of Web Services, emerging works will need to explore systems that are aware of social context of users, and are able to take into consideration their constraints and logistics factors [Di Nitto, et al., 2008]. Future directions will include a deeper understanding of how human user behaviour within a local context affects the global collective behaviour of systems. Engaging of large numbers of distributed users, involving interdependent work, remains a challenge, particularly in modelling global collective behaviour [Kearns, 2012].

Web Services have been concerned with the representation of real world knowledge to enable a meaningful discovery of Web Services for the task at hand. Despite the availability of manually encoded and automated discovery of semantic collections, we are still challenged in terms of meaning-aware applications.

A major challenge with Semantic Web Services lies in the integration of ontologies across applications with very little agreement on vocabulary and shared concepts. Finding common concepts across semantic databases such as Cyc, YAGO, DBpedia and Geonames is not a trivial feat. Not only do these repositories differ in concept representation, they also present contradictory and conflicting information [Kulathuramaiyer et al., 2014] The question is not only to come up with a unified set of reference concepts that can be mapped across Linked Open Data schemas [Bergman \& Giasson, 2014]; we need to address the notion of truth as discussed in [Kulathuramaiyer, et al., 2014]. Even for simple concepts like 'tallest mountains' there can be a variety of interpretations.

The rest of the paper will then mainly focus on the concerns of the web ecosystem and its immense implications on our lives.

\section{Concerns}

The discussions so far has indicated numerous areas of concerns relating to emerging web technology and applications. As we want the web to be able to help humanity, we take a broader view of developments. We revisit Figure 4, but this time reviewing the concerns arising from it. Figure 5 subsequently highlights the problems at each level based on the previous discussions. Development on the web to a large extent has been ad-hoc and loosely coupled in addressing concerns of structure and growth, particularly when problems are encountered, and at times in a patchy way. In this respect, in Section 4.1 we present a discussion on transclusion.

Profiling and the quality of judgment by rating services are areas that can benefit from a more human-centric efforts. We need to address issues such as privacy and exploitation of efforts 


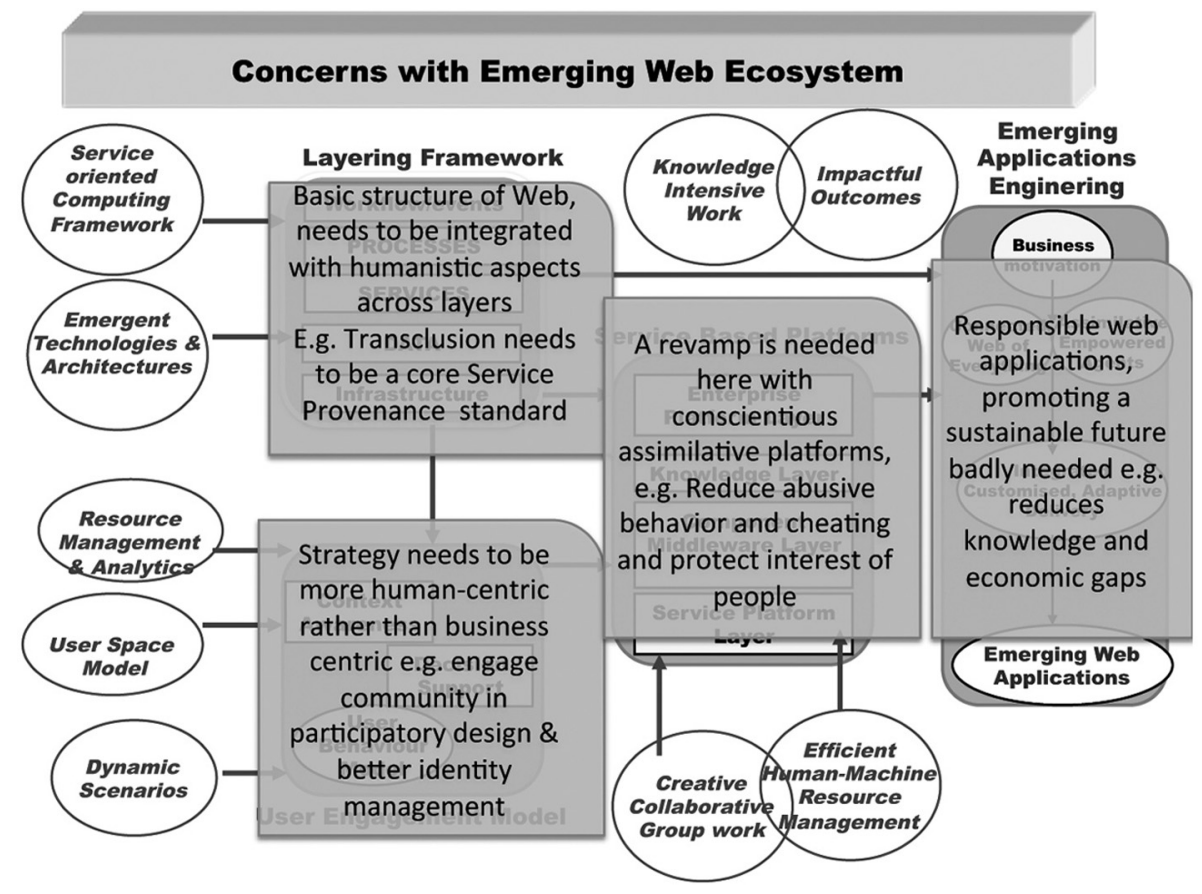

Figure 5. Overview of emerging web ecosystems.

mentioned in the previous Section. As a variety of forms of infringements are rising on the web, there is a need to look at fundamental issues such as the notion of truth and the question of reliability. The reliability of information is still a major concern. At the same time, broader social and political concerns cannot be overlooked. We need to examine both the micro and macro level dimensions in harnessing the inseparable interaction between human and non-human actors [Halford, et al., 2010].

\subsection{Transclusion and Concerns on Structure of the Web}

Transclusion describes an advanced way for including documents or parts thereof within the context of other documents, without an actual need for duplicating the original document. Though this approach was originally proposed by Ted Nelson [Nelson, 1965], this pioneering idea has not been fully exploited. Transclusion usually takes the form of a citation, whereby the source of the document is clearly maintained while providing a visible link to the source. Implementation aspects with regard to transclusion have been described in [Krottmaier \& Maurer, 2001; Maurer \& Kolbitsch, 2006].

A transclusion system, incorporated within the Austria Forum ${ }^{10}$ since 2008, serves as a demonstration of this powerful feature. It is possible to incorporate an entire page or segments of it within any Wiki page on Austria Forum. The content of a page will be displayed at run-time within another Wiki page, without having to duplicate contents. There is, however, a restriction in the current implementation as the specification of sections on the original page (by a transcluding author) can only be done with edit permissions on the original page. Despite this limitation, this feature has made it much easier for content management across the various knowledge sources in Austria Forum.

The value of such a system is in the ability to track the access to all transcluded components directly without imposing additional cognitive loads on the users. A tracking mechanism would allow automatically mechanisms for copyright management and micro-payment [Nelson, 1997] whereby the original authors could be rewarded for their original works.

When users perform copy-paste, the link to the original context of the source document is lost.

\footnotetext{
${ }^{10}$ http://austria-forum.org
} 
Such a situation could be avoided if transclusion was in place with its widespread use. Transclusions would replace all the copy-and-paste done on the web today, via an automated management system for re-using ideas in a legal way.

Transclusion structure would help to overcome many of the problems that we are still struggling with on the web. Apart from its use in authoring and publications systems, micro-payments and intellectual property management, it would enable information discovery systems, on-thefly insertion, content repurposing and dynamic adaptation for a variety of application such as eCommunities and electronic encyclopedia and knowledge management systems [Kolbitsch \& Maurer, 2006].

\subsection{Notion of Truth and Reliability of Information}

Information providers play an important role in shaping the reality for millions of users. The billions of daily searches have severe implications on the lives of people. As many new users become fully trusting of the rapid answers that the web is able to provide, their influence expands even further. Helpful services like auto-complete suggestions ${ }^{11}$ have become a powerful medium that intervenes strategically during the intention-specification stage of web searchers. In this process this service guides the articulation of intentions leading to fast and easy prediction of 'relevant answers'.

The web is thus producing a new form of meaning creation [Baker \& Potts, 2013] by observing the behavior patterns of users in which they are able to influence directly the search process of users. Search and ranking algorithms are emphasizing subjective relationships based on partial notions of truth as discovered from usage patterns. E.g. there have been situations where degrading associations were assigned to particular user names [Baker \& Potts, 2013] by basing entirely on hypes and rumours [Niggemeier, 2012]. Such a service can easily be further exploited for performing a targeted character assassination of individuals.

Ways to prevent users from consuming and inadvertently contributing to information that constitute partial truths of non-diligent sources needs to be explored. The notion of truth needs to be re-emphasized and 'discovery of truth' needs to once again become a core activity.

Acquiring reliable knowledge and inquisitive questioning of truth are pre-requisite core research skills for all learners and learning communities. Information providing sites should explore ways of engaging communities in scholarly pursuits that helps to nurture these traits. Responsible information sources should be distinguished and recognized for providing alternative measures of reliability or by providing exploratory directions [Maurer, 2014]. In any case, the source of information has to be duly considered, scrutinized and made known to users. Information providing sites should therefore take into consideration the different needs of users, rather than propagate popular directions only.

Being particularly motivated about the perception of truth is a characteristic that cannot be compromised, particularly not when it comes to scholarly activities. This motivation, which closely relates to inquisitiveness and passion for knowledge, is now being replaced by an acceptance of stereotypical ideas and the focusing on the trivialities as propagated by social media through repeated posts and reposts. As users are being left in a distracted state, we do have a daunting task ahead.

\subsection{Reliabilty of Information and Services}

The reliability of information on the web has been seen to be questionable for a variety of reasons. Limitations identified in previous works include: erroneous, incomplete; slanderous and contradictory information [Maurer \& Kulathuramaiyer, 2009], identity fraud [Kolbitsch \& Maurer, 2006], the spread of lies, scandals, rumour mongering [Keen, 2007] and biased, skewed [Eom, et al., 2014] or contentious information [Sumi, et al., 2011], [Yasseri, et al., 2014] [Weissman et al., 2014]. As there may be factual drifts with misleading claims, determining the notion of truth then becomes a challenge. Though professional scholars may not be affected by the skewness, students and public can be affected significantly [Eom, et al., 2014].

\footnotetext{
${ }^{11}$ Google. Google autocomplete http://support.google.com/websearch/bin/answer.py?answer=106230
} 
Readers of contributors in open web encyclopedias such as Wikipedia, have no guarantees, whether the contents are objective, complete or what it says is still applicable [Maurer, 2014].

The Austria Forum, on the other hand, explores the provision of reliable information, by first performing a verification step before information can be considered 'frozen' in time according to a context. A responsible information supply service will have to be accountable for the content that is made available to the public. The 'Geography of the World' section of the Austria Forum has presented us with numerous challenging scenarios. In the efforts to consolidate and verify information from a number of sources such as the CIA Facbook, Britanica, Worlfram Alpha, Geonames and Wikipedia, we were faced with conflicting information in many situations.

An elaborate discussion on the outcomes of the verification tasks can be found in [Kulathuramaiyer, et al., 2014]. We compared and contrasted the information across these various sources for quantitative questions like: What is the size of Sweden? Which is the largest city in Canada? In situations where we found reasons for the conflict, we were able to display the correct information, while making known the basis for conflicts to arise. In situations where we did not know the reasons for the conflicting results, we presented alternative answers, while stating the source to users. We then made a plea to the community to provide reasons for the discrepancies in data.

We illustrate the notion of truth with regard to reliability of information propagated by the following example. In determining the most important persons in history, Wikipedia has been shown to produce surprising results. For example, Carl Linnaeus the $18^{\text {th }}$ century botanist, has been determined to be the most important person in history even more that Jesus, Hitler or Michael Jackson [Powell, 2014]. Despite difference in ratings, according to different ranking schemes [Rotberg, 2014], it has also been shown that important historical figures were those who were mainly male, born in Western countries after the $17^{\text {th }}$ century [Eom, et al., 2014]. By basing entirely on the PageRank or Inlinks al- gorithms, a true reflection of reputation cannot be conclusively established.

As a key step in checking reliability, [Kulathuramaiyer, et al., 2014] checked the reliability of basic quantitative information that are normally taken for granted. Our findings show that there were discrepancies in the information provided, across the different knowledge sources. This makes it difficult to identify the correct answers or determine the truth behind these assertions without a deliberate research effort on the part of users.

The question of whose responsibility it is to protect the interest of vulnerable groups from the spread of potentially dangerous sentiments or beliefs (or a mixture of truths with camouflaged partial truths) is yet to be resolved. Issues of reliability of information, the accountability for providing them and the resulting consequences have to be studied and carefully addressed.

Web sources largely use aggregation services of unknown quality or gather data from un-trusted sources. Some of these information may be true with regard to a particular culture or point of view or a particular context. Others may be merely a belief, a superstition a joke or a rumour. In order to promote a deeper understanding based on a specific point of focus, there should be regional servers that either focus the aggregation according to locality or are based on culturally sensitive common notions and understanding. In this respect, information services are emerging at the regional or community levels such as Austria Forum and $\mathrm{Su}-\mathrm{Si}^{12}$, to address some of these concerns within localized contexts, taking a regional perspective.

Another proposal that can be employed in the integration of life-long-learning modules within eCommunities is to engage the community to deliberate on the notions of truth and become aware of different points of view. The power of mass collaboration systems will also need to be used to address this in a meaningful way.

\subsection{Social Network Fatigue}

Studies from Pew Internet and American Life Project [Van Grove, 2013] and GlobalWebIndex

12 http://www.su-si.at/ 
[Sawers, 2012] indicate that the 'Facebook Fatigue' is real and is actually affecting web users.

The notion of fatigue with the use of social networks can be illustrated by reviewing the layered view of eCommunities as seen in Figure 3. When users get trapped in a continuous engagement with a large group of friends and pseudo-friends beyond a manageable threshold, these networks can pose severe strains on individuals. This can also be aggravated further if users do not have a support clique at the inner core eCommunity layer. The large amount of time spent in maintaining relationships with a large mixed group of connections, apart from dealing with intrusively interspersed invitations and advertisements and ingeniously infiltrating spammers can be extremely energy-draining.

As the separation between work and play disappears, casual discussions may be used by authorities for purposes that one may not foresee. Social networks in this way are causing undue stress on many individuals. It is thus not surprising that many users end up taking a break at least once from their tiring and at times stressful Facebook membership.

\subsection{Affecting Learner Experience}

The book by Nicholas Carr, 'The Shallows' described the works of scientists in Stanford University, highlighting that 'the Internet is promoting cursory reading, distracted thinking and superficial learning' [Carr, 2010]. Users are however considering themselves to be much smarter than ever before as they can cope with larger amounts of data. They are consequently reading much less than ever before [Carr, 2010], with serious reading becoming a challenge. As people are thus using mainly the part of their brain that deals with short-term memory, they are not able to cope with the increasing cognitive loads [Carr, 2010]. The engagement with social media ties the users' focus and attention on a particular problem solving situation, with a locked-up sensory perception.

Studies also show that our brain has limitations on the number of stable friends that we can maintain on Facebook [Reisinger, D., 2010] or other social media sites [Goncalves, etl. Al.,
2011]. The number of active connections handled is also limited by the cognitive ability of our short-termed memory [Reisinger, D., 2010]. As the number of pseudo and superficial friends increases together with needs to multi-task across social obligations, users gradually become unable to focus their attention span to commit to the rigour and discipline needed for deep reflection.

The situation is aggravated by the amount of distractions that is increasingly calling for the immediate and instant servicing the competing feeds of presumed importance. As we become oblivious of the real world around us [Carr,2010], users get more and more sucked into a vicious circle. The web, together with new media, has worsened the quality of life in many ways as the emerging channels of media tend to be far too distracting, causing people to become far less focused [Weber, 2006].

The web is also encouraging a horizontal modelling that relates to the mimicry of peers and people of the same age resulting in the communication and alarming knowledge gaps in vertical modelling with respect to interactions with older people [Bauerlein, 2008] Here we see a social danger whereby teens chose to compulsively interact with digital technology and become unable to connect with the older generations. They get caught up with the need to be inline with latest developments within cliques and in this way continuously enhance and stabilize popularity. The protective older outlook then becomes totally irrelevant.

We illustrate this arising situation with the statistics presented by GuardChild, which is a site that continuously collects data on internet use from numerous studies. While $96 \%$ of teens have access to social network sites, only $15 \%$ of the parents know about their children's cyber socialising habits ${ }^{13}$.

\subsection{Culture of Mediocrity}

Being inundated with information affects our cognitive power. As a result, our power of reflection suffers. This, in conjunction with the constant distractive force, deters insightful thinking and creative work [Weber, 2006], [Carr,

\footnotetext{
${ }^{13}$ http://www.guardchild.com/statistics/
} 
2010]. The distractive force is not easy for us to deal with as our sensory experiences have been conditioned to trap us in clamoring for a 'neverending state' of media engagement. As users lose their ability to distinguish relevant information and the ability to understand subjects, their learning remains shallow [Carr, 2010]. As a large enough number of users lose their ability for distinguishing relevant information and 'glorify' their shallow learnings, the culture of mediocrity emerges.

Superficial learning and fast-and-easy publications then become widespread, having compromised the rigorous process of learning through discovery. This not only endangers the future of creative work; it also destroys future generations through a culture of mediocrity [Weber, 2006].

\subsection{Privacy Still Needs to be Preserved}

Can we ignore privacy concerns completely? Can we just entirely accept the total compromise of privacy and just remain unaffected? The Circle [Edgers, 201] warns us against the "continuous striving for perfection' in the course of 'compromising freedom'. The novel sheds insights on the emerging life-styles and the undesirable situations that are produced via the commitment of users through the entanglement in this vicious circle. The dehumanising compromises that we make cannot be taken lightly. Naive practices of private information exchange for gaining appreciation through 'likes', and increased following bonds us to a culture of wanting to share more, with the desire to know everything about everybody. Life becomes one that puts us in a vigilant state, as we know we are being watched all the time. Furthermore, once information is captured on the web, it will remain there for eternity. It also becomes a norm and an open culture where information can be mined and used outside its original context.

The acceptance of users to such a lifestyle in response to services received is seen in subscriptions to new web services like 'Patients like me' where users are willing to share personal information rather easily. Even when we trust sites providing such services, users tend to ambivalent about the potential threats [Kelly, S.M., 2014] of leakages in a post Snowden era.
As pointed out by [Nussbaum, 2007], the way the uninhibited future generation are taking to the web, openly discussing anything and everything, warns us about the the alarming future where privacy may not be relevant anymore. Most users are moving towards acceptance or ambivalence of these concerns [Zimmer, 2008].

\subsection{Reputation, Rating Services and User Profiling}

Reputation is often established using mass collaborative systems to aggregate and classify users based on generated contents and browsing behaviour. Reputation is normally measured by the number of followers, likes, views or reblogs. Other measures include abstract user ratings, measures such as mentions, sentiments, passion, and reach [Kietzmann, et al., 2011].

Reputation is also closely related [Kietzmann, et al., 2011] to notions of identity, conversations, sharing, presence, relationships and groups. Predictions that are made by recommender or rating systems are mainly based on the perceived identity of users, based on the transactions such as presence, relationships, participating groups, and sharing and conversation activities. Based on the rating or rankings of users, judgments are made on profiles and identity.

Collective human judgment is used as a basis for ranking of sites, services and information clips or media units. These rating services are capable of providing profiling 'truth' ratings that can be subject to malicious activities, multiple identity attacks and the orchestrated ratings by buying in user votes [Molavi Kakhki, et al., 2013]. As progress into these research areas continues, steps need to be taken in engaging the users and motivating them to consider more seriously the accuracy of perceived truth notions.

Rating systems need to manage users in a dynamic way. Productive users need to be identified, given more meaningful roles, with their contributions evaluated and performance ranked in determining trust level assigned to them [Doan et al., 2011]. Trusted users may then be further assigned higher engagement roles such as resolving conflicting issues or even merge conflicting inputs or supply inference rules [Kolbitsch \& Maurer, 2006], [Doan et al., 2011]. 
Owing to the current state of intelligent filtering, profiles constructed tend to be aggregated, generalized (in some ways over-generalised) and reflecting the predominant groups of users based on locality or past browsing behavior. Though we know that a deeper profiling capability is needed based on humanizing attributes, the protection of data and the accountability for the data needs to be in place.

\subsection{Emerging Trends}

The blurring of roles between experts and amateurs, virtual and real worlds, and play and work have led to new types of jobs, game-changing industries, symbiotic man-machine relationships and many other developments that are changing our life-styles. In the pipeline, we see many developments that are poised to disrupt age-old industries. Examples of emerging startups are already affecting the service industries such as transportation, hospitality, food service, staffing and talent, office rental, banks, public relations and many others [Hinchcliffe, 2014]. Some of these developments include the crowdenabled sharing of office-space or specialised equipment, on demand access to cars and on line market-place of staffing. Further discussions on web applications will be dealt with in another paper in this issue.

The flattening of culture and leveling of expertise [Keen, 2006] is now promoting many new job roles such as citizen journalists, citizen musicians, videographers [Zimmer, 2008], citizen photographers, citizen actors, citizen lecturers, among the many emerging roles. There are also many new positions that are emerging and likely to emerge such as data scientists, ethical hackers, cyber-surveillance officers and citizen trends analysts.

The issues on what needs to be taught to future generations needs to be reviewed and scrutinized. What we are now teaching in schools involves, to a large extent, the memorization of facts which could be gathered by students by accessing the various media channels becoming available. Focus should however be emphasizing advanced information literacies and social learning skills.

There are also a number of unintentional consequences of emerging developments that cannot be overlooked. These include deeper global controls, increase in crime and infringements, prevailing commoditized viewpoints and the widening gaps and exploitation.

There are new types of violence and infringement that arise with emerging technological developments. Terms like cyber violence, cyber bullying, cyber safety, active by-stander, cheat engines can be viewed as indicators of emerging trends. Just as the web can be used for education purposes to prevent violence or in displaying concerns to help promote safety, it can also directly or indirectly promote violence. As television is widely attributed to increase, diversification and democratization of violence and crimes, there is a need to be wary of even more powerful influence of the web on our future generations. There have been concerns relating to video clips that have incited violence on video-sharing sites, by inadvertently arousing the sentiments of users. Although these clips have been removed by sites such as YouTube for some regions, when such situations happen, the damage on innocent lives cannot be undone.

In the same way, the web can also be seen to be promoting cheating and other unethical practices. As an illustration of what users search for is revealed by the auto-complete suggestions, as shown in Figure 6. Obviously, the web has been used for cheating and also in exploring ways to prevent it when the problem occurs. An interesting point to note is that the directions (based on Google search suggestions) that indicate the path taken by those who intend to commit an act, can also be used by those who want to help. The main concern here is that, without proper education, young minds tend to become influenced by negative tendencies much too easily, leading to many social problems.

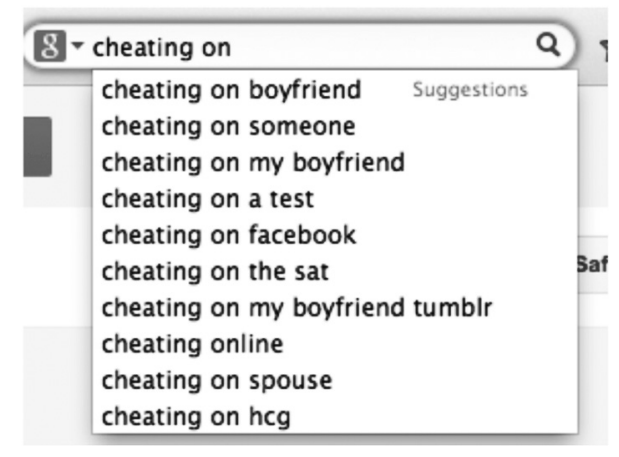

Figure 6. Search results indicating trends. 
As nothing is unanimous on the web, companies such as Google can help project all sorts of visualisations, while having the potential to identify those who are guilty. These global companies could definitely provide private investigator services aside from providing information to governments.

Anonymous identities have also been noted to be a reason for encouraging cyber-bullying, including committing of off-topic and off-color comments [Kietzmann, et al., 2011]. Whether anonymity in terms of not specifying names or not being registered should be condoned is an issue to be dealt with by mass collaboration systems and eCommunities.

\subsection{Macroscopic Dimensions: Inclusive Access and Participation}

The distortion of reality via restricting and manipulating user perception as discussed earlier is indirectly capable of altering the recording of history [Witten et al., 2007]. Such an ability by private companies with no specified controls on accountability can be seen to be extremely dangerous. Personalization in some way has been the cause for restricted access to users. We illustrate the restricting of user perception that arises from personalisation algorithms through a discussion on Filter Bubbles.

Filter Bubbles [Paiser, 2011], describes the filtering of information that is applied on users based on their profiles built upon search history, and other attributes compiled by search engines or social media sites. The saved profile can then be used to constrain and hide information, based on the perceived needs of a user profile. This is however being seen to impact upon the ability of individuals to discover new ideas.

The consolidated profiling of services such as Google Accounts, should in principle be expected to serve user information needs much better. Google for instance makes use of cookies from DoubleClick and Google Analytics, together with widgets from YouTube and Google+ for keeping track of user activities [Roesner, et al., 2012]. The over-generalization based on locality or past search behaviour tends to be a major delimiting factor.

The propagation of cookies across the web also serves to publish browsing patterns of users for performing third-party tracking. Profiling behaviour is affected by Filter Bubbles in many more sites than we may be aware of. A study on how researchers at Princeton are tracking the way websites discriminate against users on price and access to certain services, has been illustrated in [Olson, 2013].

User profiles constructed within a commodity culture and shaped according to sites visited is being used to determine the personalized level of quality of service. The democratic access to all resources on the web is becoming an ideology used to restrict access to unaware users within filter bubbles.

Tools and data that shape the reality for billions of people are now at the hands of large corporations and governments. As business model need to know as much as possible about each person, large corporations and governments are stretching themselves to mine deeper intrinsic patterns of users. There is a need to be concerned about the fact that data that is shaping our reality is now being held by private companies. Without a guarantee against accidental leaks, and the evolving definitions of privacy and personal data control, caution is needed.

In the arena of politics, political parties are now employing a collection of technologies for increasing the level of political participation and degree of engaged citizenship. This, combined with data analytics tools, makes these campaigns a cyber-battle of buying-in citizen willingness and commitment to co-produce the campaign buildup. [Nielsen, 2011] describes the extensive use of numerous social media tools for political mobilization, where co-production of citizenship is seen as the key to mobilize citizens.

Vast amounts of personal information go beyond the use for surveillance purposes. This can be used as the basis for exercising social disciplinary powers [Zimmer, 2008], as seen in global companies required to provide information to government in court cases.

The Net Delusion [Morozov, 2011] describes the popular belief that social network sites will bring about a cyber utopian world by the liberating power of technology. For example, the use of tools such as Twitter was expected to bring about a revolutionary break-through against authoritarian regimes. This ideology has however 
been shown to be mis-guided as these regimes have in themselves mastered technology. They use technology extensively in enforcing censorship, surveillance and propaganda. Technology is seen here as benefiting both democracies, and authoritarian regimes in curbing antigovernment movements.

\section{Conclusion}

As the web is assimilated and expands its role, we need to learn to effectively apply it to enrich our lives. At the same time, the dangers associated with this technology need to be minimized by deliberate efforts on the part of the enforcement agencies, including the users of the system. We need to prevent the abuse or misuse as much as we can. Users should also be made aware of the social and political consequences, making the democratic web actually work for all of us. The success of such efforts in making the web actually work needs rethinking with deliberations to check on consequences of emerging technologies and applications. New balanced model that does not dehumanize, need to be devised. This survey has touched on a broad range of topics within the theme, and has provided many leads for subsequent explorations.

\section{References}

[1] P. BAKer, A. PotTs, Why do white people have thin lips? Google and the perpetuation of stereotypes via auto-complete search forms, Critical Discourse Studies, 10(2), pp. 187-204, 2013, DOI : $10.1080 / 17405904.2012 .744320$.

[2] V. BARASSI, E. TrerÉ, Does Web 3.0 come after Web 2.0? Deconstructing theoretical assumptions through practice. New media \& society, 14(8), pp. $1269-1285$

[3] M. BAuerlein, The dumbest generation: How the digital age stupefies young Americans and jeopardizes our future (or, don't trust anyone under 30). Penguin.

[4] M. K. Bergman, UMBEL: A Subject Concepts Reference Layer for the Web, Slideshare, July 16, 2008, http://www.slideshare.net/mkbergman/ umbel-a-subject-concepts-referencelayer-for-the-web

[5] T. Berners-LeE, J. HendleR AND O. Lassila. The Semantic Web, Scientific American, 284(5), pp. 34-43, 2001.
[6] Bitcoin, A Peer-to-Peer Electronic Cash System bitcoin . org. October 2008.

[7] C. BLACKMORE, Landscapes of practices: social learning systems and rural innovation. In Proceedings of the IFSA Annual Symposium and Exhibitio, 2012.

[8] N. CARR, What the Internet is doing to our brains. WW Norton \& Company, 2010.

[9] Internet of Things, Cisco Internet Bussiness Solution Group, 2011. http://share.cisco.com/ internet-of-things.html

[10] N. Couldry, Media and Symbolic Power: Extending the Range of Bourdieu's Field Theory. Media@lse Electronic Working Papers Department of Media and Communications, LSE No. 2, 2003. http://www.Ise.ac.uk/collections/media@ lse/Default.htm

[11] E. Di Nitto, C. Ghezzi, A. Metzger, M. PapaZOGLOU, K. POHL, A journey to highly dynamic, self-adaptive service-based applications. Automated Software Engineering, 15(3-4), pp. 313-341, 2008.

[12] A. DOAN, R. RAMAKRISHNAN, A. Y. HALEVY, Crowdsourcing systems on the world-wide web. Communications of the ACM, 54(4), pp. 86-96, 2011.

[13] Y. H. EOM, P. ARAgón, D. Laniado, A. KaltenBRUNNER, S. ViGNA, D. L. SHEPELYANSKY, (2014). Interactions of cultures and top people of Wikipedia from ranking of 24 language editions. arXiv preprint arXiv:1405.7183.

[14] M. Georgescu, D. D. Pham, C. S. Firan, U. GadiRAJU, W. NEJDL (2014, June). When in Doubt Ask the Crowd: Employing Crowdsourcing for Active Learning. In Proceedings of the 4th International Conference on Web Intelligence, Mining and Semantics (WIMS14) pp. 12, ACM.

[15] D. GILLMOR, We the media: Grassroots Journalism by the People, for the People. O'Reilly Media, Inc. 2006.

[16] B. Goncalves, N. Perra, A. Vespignani, Validation of dunbar's number in twitter conversations. arXiv preprint arXiv:1105.5170, 2011.

[17] S. Halford, C. Pope, L. CARr, A Manifesto for Web Science?, 2010.

[18] D. Hull, K. Wolstencroft, R. Stevens, C. Goble, M. R. Pocock, P. Li, T. Oinn, Taverna: a tool for building and running workflows of services. Nucleic acids research, 34(2), pp. W729-W732, 2006.

[19] D. HinchClIFFE, What is the future of work, On Web Strategy, http://dionhinchcliffe.com /2014/03/27/what-is-the-future-of-work/

[20] A. Iera, G. Morabito, L. Atzori, The Convergence of Social Networks and the Internet of Things: Opportunities, Technologies and Challenges, 2014. IEEE World Forum on Internet of Things (WF-IoT), 6-8 March 2014 - Seoul, South Korea. 
[21] M. IngRAm, Two charts that tell you everything you need to know about the future of newspapers, Apr. 11, 2013. https://gigaom.com /2013/04/11/two-charts-that-tell-youeverything-you-need-to-know-about-thefuture-of-newspapers/

[22] S. M. Kelly, How to Protect Your Photos (Nude or Otherwise) From Hackers on iCloud, http://mashable.com/2014/09/01/icloudnude-photo-hack/

[23] A. KeEn, Web 2.0: The second generation of the Internet has arrived. It's worse than you think, Daily Standard, 15 February, 2006. http: //www. weeklystandard.com/Content/ Public/Articles/000/000/006/714fjczq. asp

[24] A. KEEN, The Cult of the Ametuer: How Today's Internet is Killing Our Culture, Doubleday/Currency, New York, 2007.

[25] M. KEARNS, Experiments in social computation. Communications of the ACM, 55(10), pp. 56-67, 2012.

[26] A. Kittur, J. V. Nickerson, M. S. Bernstein, E. M. Gerber, A. Shaw, J. Zimmerman, M. Lease, J. J. Horton, The future of crowd work. In Proceedings CSCW 2013, ACM Press, pp. 1301-1318, 2013.

[27] A. Kittur, B. Smus, S. Khamkar, R. E. Kraut, CrowdForge: Crowdsourcing complex work. In Proceedings UIST 2011, pp. 43-52, ACM Press, 2011.

[28] J. H. Kietzmann, K. HeRmKens, I. P. MCCARThy, B. S. SILVESTRE, Social media? Get serious! Understanding the functional building blocks of social media. Business horizons, 54(3), pp. 241-251, 2011.

[29] J. Kolbitsch, H. A. MAURER, The Transformation of the Web: How Emerging Communities Shape the Information we Consume. Journal of Universal Computer Science, 12(2), pp. 187-213, 2006.

[30] H. Krottmaier, H. A. Maurer, Transclusions in the 21st Century. Journal of Universal Computer Science, 7(12), pp. 1125-1136, 2001.

[31] N. Kulathuramaiyer, W. T. Balke, Restricting the View and Connecting the Dots: Dangers of a Web Search Engine Monopoly, Journal of Universal Computer Science, 12(12), pp. 1731-1740, 2006. http: //www. jucs .org/jucs_ 12_12/restricting_the_view_and

[32] N. Kulathuramaiyer, H. Maurer, Learning ecosystems for dealing with the copy-paste syndrome. Journal for Research in Innovative Teaching, 1(1), pp. 1-25, 2008.

[33] N. Kulathuramaiyer, H. Maurer, Why Elearning as it stands is not enough. In Rainbow of Computer Science, Springer Berlin Heidelberg, pp. 157-164, 2011.
[34] V. LID, Why socialising doesn't scale, on February 4, 2010. http://blog.viil.net/2010/02/whysocializing-doesnt-scale/

[35] T. Matranga, Shared publicly, Dec 1, 2013. https://plus.google.com/+ TancrediMatranga/posts/BeUSdezcMpU.

[36] H. Maurer, J. Kolbitsch, Transclusions in an html-based environment. CIT. Journal of computing and information technology, 14(2), pp. 161-173, 2006.

[37] N. Kulathuramaiyer, H. MaURER, Coping With the Copy-Paste-Syndrome. In Proceedings of World Conference on E-Learning in Corporate, Government, Healthcare, and Higher Education, 2007, pp. 1072-1079, AACE.

[38] H. MAurer, Austria Forum and Beyond, $10^{\text {th }}$ May, 2014. http: //austria-forum.org/attach /Crowdfunding/14-05-10-Future-ofAustria-Forum.pdf

[39] H. MAURER, Is the Internet turning us in dummies? Communications of the ACM, 58(1), pp. 48-51, 2015.

[40] A. Molavi KakhKi, C. Kliman-Silver, A. MisLOVE, Iolaus: Securing online content rating systems. In Proceedings of the 22nd International Conference on World Wide Web, pp. 919-930, 2013.

[41] S. NiggemeIER, 'Autocompleting Bettina Wulff: Can a Google Function Be Libelous?' Spiegel, September 20, 2012. http://www.spiegel.de /international/zeitgeist/googleautocomplete-former-german-first-ladydefamation-case-a-856820.html

[42] T. H. Nelson, A File Structure for the Complex, the Changing and the Indeterminate, Proceedings of the ACM 20th National Conference, Cleveland, OH, U.S.A., pp. 84-100, 1965.

[43] T. H. NELSON, Transcopyright: Pre-permission for virtual republishing, 1997.

[44] J. NIELSEN, Digital Divide, The Three Stages. Jakob Nielsen's Alertbox, November 20, 2006. http: //www. useit. com/alertbox/digitaldivide.html

[45] J. NIELSEN, Participation Inequality: Encouraging More Users to Contribute. Jakob Nielsen's Alertbox, October 9, 2006. http://www.useit.com/ alertbox/participation_inequality.html

[46] R. K. NIELSEN, Mundane internet tools, mobilizing practices, and the coproduction of citizenship in political campaigns, New Media \& Society, 13, pp. 755-771.

[47] E. Nussbaum, Kids, the Internet, and the end of privacy. New York Magazine, February, 2007. at http://nymag.com/news/features/27341/ 
[48] P. OLSON, This Landmark Study Could Reveal How The Web Discriminates Against You. Forbes, 2, February, 2013. http: //www . forbes . com/sites /parmyolson/2013/12/02/this-landmarkstudy-could-reveal-how-the-webdiscriminates-against-you/?utm_campaign =techtwittersf\&utm_source=twitter\&utm medium=social

[49] E. PARISER, The filter bubble: What the Internet is hiding from you. Penguin UK, 2011.

[50] R. Powell, Sydney Morning Herald, Digital Life, Wikipedia's most influential people: Carl Linnaeus, Jesus, Hitler, Michael Jackson, June 11, 2014. http://www.smh.com.au/digitallife/digital-life-news/wikipedias-mostinfluential-people-carl-linnaeus-jesushitler-michael-jackson-20140611zs39r.html

[51] D. REISINGER, Sorry, Facebook friends: Our brains can't keep up. Cnet.com, January 25, 2010.

[52] R. I. RotBERG, The Algorithms of Fame. Journal of Interdisciplinary History, 45(2), pp. 209-217, 2014.

[53] T. Kohno Roesner, D. Wetherall, Detecting and Defending Against Third-Party Tracking on the Web. NSDI, 2012.

[54] P. SAWERS, Facebook fatigue is spreading but social media is on the rise, says Internet study. TheNextWeb, 6 February, 2012. http://thenextweb .com/socialmedia/2012/02/06/facebookfatigue-is-spreading-but-social-mediais-on-the-rise-says-internet-study/

[55] M. W. Steen, P. Strating, M. M. Lankhorst, H. TER DOEST, M. E. IACOB, Service-oriented enterprise architecture. Z. STOJANOVIC, A. DAHANAYAKE, Service Oriented Systems Engineering, Hershey, pp. 132-154, 2005.

[56] R. Sumi, T. YAsseri, A. Rung, A. Kornai, J. KERTÉSZ. Characterization and prediction of Wikipedia edit wars. Proceedings of the ACM WebSci, bf 11, pp. 1-3, 2011.

[57] J. TraVERS, S. Milgram, An experimental study of the small world problem. Sociometry, pp. 425-443, 1969.

[58] Union Metrics, TweetReach blog, January 14, 2013. https://blog. tweetreach.com/2013/01/ checking-in-on-the-2013-golden-globeson-twitter-and-tumblr-what-did-fansthink/

[59] J. VAN DIJCK, Facebook as a tool for producing sociality and connectivity. Television \& New Media, 1527476411415291.

[60] J. VAN Grove, Study: Facebook Fatigue is Real. CNet.com, February 5, 2013.

http://www. cnet.com/news/studyfacebook-fatigue-its-real/

[61] S. WEBER, Das Google-Copy-Paste-Syndrom, Wie Netzplagiate Ausbildung und Wissen gefährden. Heise, Hannover, 2006.
[62] I. H. WitTen, M. Gori, T. Numerico, Web Dragons. Inside the Myths of Search Engine Technology, Morgan Kaufmann, San Francisco, 2007.

[63] E. WENGER, Social learning capability: four essays on innovation and learning in social systems. Social Innovation, Sociedade e Trabalho. Booklets 12 separate supplement, 2009, MTSS/GEP \& EQUAL Portugal, Lisbon.

[64] E. Wenger, Communities of practice and social learning systems: the career of a concept. In Social learning systems and communities of practice, $\mathrm{pp}$. 179-198, Springer London, 2010.

[65] S. Weissman, S. AYHAN, J. BRADley, J. Lin, Identifying Duplicate and Contradictory Information in Wikipedia, arXiv preprint arXiv, pp. 1406-1143, 2014.

[66] T. YASSERI, A. SPOERri, M. GRAHAM AND J. KERTÉSZ, The most controversial topics in Wikipedia: A multilingual and geographical analysis. In: P. FICHMAN, N. HARA, editors, Global Wikipedia: International and cross-cultural issues in online collaboration, Scarecrow Press, 2014.

[67] T. Zaman, A. W. YeO, N. KulathuramaiYer, Augmenting indigenous knowledge management with information and communication technology. International Journal of Services, Technology and Management, 19(1-3), pp. 137-148, 2013.

[68] C. Zhong, M. Salehi, S. Shah, M. Cobzarenco, N. SASTRY, M. CHA, Social bootstrapping: how pinterest and last. fm social communities benefit by borrowing links from facebook. In Proceedings of the 23rd International Conference on World Wide Web, (pp. 305-314), April 2014. International World Wide Web Conferences Steering Committee.

[69] M. ZIMMER, The externalities of Search 2.0: The emerging privacy threats when the drive for the perfect search engine meets Web 2.0. First Monday, 13(3). http: //firstmonday.org/htbin/ cgiwrap/bin/ojs/index.php/fm/article /viewArticle/2136

Received: October, 2014 Accepted: October, 2014

Contact addresses: Narayanan Kulathuramaiyer Faculty of Computer Science and Information Technology University Malaysia Sarawak Malaysia

e-mail: nara@fit.unimas.my

Hermann Maurer Institute for Information Systems and Computer Media (IICM) Graz University of Technology Austria 
NARAYANAN KULATHURAMAIYER is currently a Professor of Computer Science at the Faculty of Computer Science and Information Technology, Universiti Malaysia Sarawak (UNIMAS). He received his Ph.D. in Computer Science from Graz University of Technology, Austria. He serves as the Director of the Web Intelligence Consortium (WIC), Malaysia Research Centre, Editor-in-Chief for the Journal of Universal Computer Science and Dean of the Faculty.

$\mathrm{He}$ is also currently a member of the National ICT Human Resource Taskforce, Steering Committee member of the National Citation Centre, member of Nations Professor Council and Fellow of Centre of Excellence on Rural Informatics, UNIMAS. He has been a part of a number of Research and Commercialisation projects which include European Commision projects, Japanese Human Resource grants and the National Language Technology Productisation project including a number of high-profile Consultancy projects.

His research interests include Semantics-Aware Systems, Knowledge Management (Governance), Technology Assimilated Learning and Web Science. He currently has over 70 refereed publications.

$\mathrm{Ph} \mathrm{D}$ in Computer Science, Graz University of Technology, Austria, 2008. Master of Science in Computer Science, Universiti Sains Malaysia, 1989. Bachelor of Computer Science, Universiti Sains Malaysia, 1988.

HERMANN MAURER Study of Mathematics at the Universities of Vienna (Austria) and Calgary (Canada) starting in 1959. System Analyst with the Government of Saskatchewan and mathematician-programmer with IBM Research 1963-1966. Ph.D. in Mathematics from the University of Vienna 1965.

Assistant and Associate Professor for Computer Science at the University of Calgary 1966-1971. Full Professor for Applied Computer Science at the University of Karlsruhe, Germany, 1971-1977, and Visiting Professor at SMU, Dallas, University of Brasilia (Brazil)and University of Waterloo. Full Professor at the Graz University of Technology since 1978, Dean of Studies and later founding Dean of the Faculty of Informatics. Director and board member of various research organisations and companies.

During sabbaticals, adjunct Professor at Denver University 1984-1988, professor for Computer Science at the University of Auckland, New Zealand 1993-1995 and visiting Researcher at Edith Cowan University, Perth, Australia in 2003.

Has received a number of awards, among them three honorary doctorates from the Universities of St. Petersburg, Karlsruhe and Calgary. Foreign Member of the Finnish Academy of Sciences since 1996 and member of the Academia Europaea since 2000 where he is currently one of the directors of the board. In January 2001 he was awarded the "Austrian Cross of Honours for Arts and Science Class I" and in July 2001 the "Large Medal of Honour of the Province of Styria".

He is author of twenty books, author of more than 700 papers, member of the editorial board of numerous journals program committees of conferences, in particular one of the founders of the conference series ED-MEDIA. He was project manager of a number of multimilliondollar undertakings including a patent for optical storage device, the development of a colour-graphic microcomputer (MUPID), electronic teaching experiments, multi-media projects such as "Images of Austria" (Expo'92 and Expo'93), of electronic publishing projects and of the development of the first second generation Web Based Information System Hyperwave; participated in a number of EU projects.

Main research and project areas: Networked multimedia/hypermedia systems; electronic publishing and applications to university life, exhibitions and museums, Web based learning; languages and their applications, data structures and their efficient use, computer networks, computer supported new media, and social implications of computers, including authoring science fiction stories and novels. 